\title{
In Vivo Evaluation of Engineered Self-Assembling Silk Fibroin Hydrogels after Intracerebral Injection in a Rat Stroke Model
}

\author{
Natalia Gorenkova, ${ }^{\dagger}$ Ibrahim Osama, ${ }^{\dagger}$ F. Philipp Seib, ${ }^{*}, \dagger, \S_{(0)}$ and Hilary V.O. Carswell ${ }^{*},, \S_{\odot}$ \\ ${ }^{\dagger}$ Strathclyde Institute of Pharmacy and Biomedical Sciences, University of Strathclyde, 161 Cathedral Street, Glasgow G4 0RE, \\ United Kingdom \\ ${ }^{\ddagger}$ Leibniz Institute of Polymer Research Dresden, Max Bergmann Center of Biomaterials Dresden, Hohe Strasse 6, Dresden 01069, \\ Germany
}

\section{Supporting Information}

\begin{abstract}
Targeting the brain cavity formed by an ischemic stroke is appealing for many regenerative treatment strategies but requires a robust delivery technology. We hypothesized that self-assembling silk fibroin hydrogels could serve as a reliable support matrix for regeneration in the stroke cavity. We therefore performed in vivo evaluation studies of selfassembling silk fibroin hydrogels after intracerebral injection in a rat stroke model. Adult male Sprague-Dawley rats $(n=24)$ underwent transient middle cerebral artery occlusion (MCAo) 2 weeks before random assignment to either no stereotaxic injection or a stereotaxic injection of either self-assembling silk fibroin hydrogels $(4 \% \mathrm{w} / \mathrm{v})$ or PBS into the lesion cavity. The impact on morbidity and mortality, space conformity, interaction with glial scar, interference with inflammatory response, and cell proliferation in the lesion cavity were examined for up to 7 weeks by a

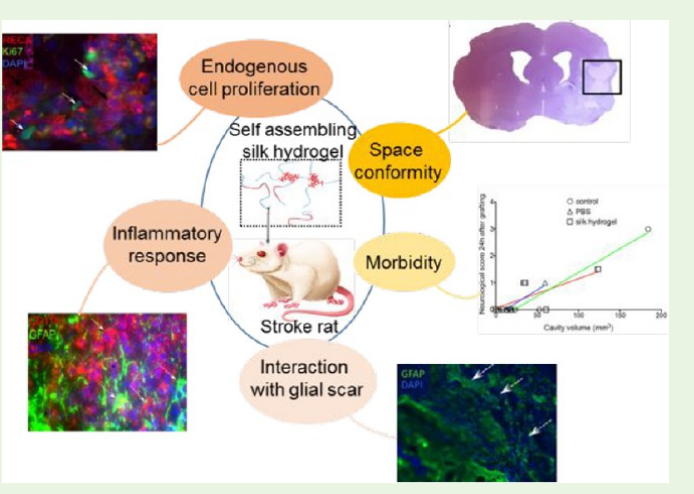
blinded investigator. Self-assembling hydrogels filled the stroke cavity with excellent space conformity and presented neither an overt microglial/macrophage response nor an adverse morbidity or mortality. The relationship between the number of proliferating cells and lesion volume was significantly changed by injection of self-assembling silk hydrogels. This in vivo stroke model confirmed that self-assembling silk fibroin hydrogels provide a favorable microenvironment as a future support matrix in the stroke cavity.
\end{abstract}

KEYWORDS: middle cerebral artery occlusion, biomaterials, silk, hydrogel

\section{INTRODUCTION}

Stroke is a leading cause of adult neurological impairment, with $20 \%$ of its survivors requiring institutionalized care; of these, up to $30 \%$ are severely and permanently disabled, with limited spontaneous recovery. ${ }^{1}$ Current strategies aimed at enhancing the limited spontaneous regenerative capacity of brain tissues include the use of small-molecular-weight drugs, biologics, and cell-based therapies. For example, growth and neurotrophic factors (e.g., vascular endothelial growth factor and brain and glial cell-derived neurotrophic factors $)^{2,3}$ improve recovery after stroke in rodent models, most likely by promoting endogenous repair by stimulating and recruiting cells. However, biologics and cell-based therapies face a major unmet challenge due to the lack of suitable methods to deliver, protect, and retain these payloads at the desired site of action. ${ }^{4}$ The lesion cavity is an ideal site for administration, as it is adjacent to the site of greatest neuroplasticity after stroke; ${ }^{5}$ however, it lacks an extracellular matrix $(\mathrm{ECM}){ }^{6}{ }^{6}$ is surrounded by a glial scar; and is filled with extracellular fluid, debris, and inflammatory mediators. ${ }^{7}$ Consequently, engineering strategies are needed to turn the lesion cavity into a microenvironment that is receptive to regenerative repair processes.
Biomaterials are ideally placed to serve this function. ${ }^{8,9}$ For example, biomaterials can replace the lost $3 \mathrm{D}$ microenvironment by physically filling the lesion cavity, mimicking brain ECM and supporting endogenous repair mechanisms. ${ }^{10,11}$ They can also serve as a potential delivery matrix for therapeutics payloads. ${ }^{12}$ To date, several studies have shown encouraging results following the use of synthetic biomaterials in preclinical models of stroke. ${ }^{4}$ However, currently used biomaterials, such as synthetic hydrogels, typically require chemical cross-linking, which necessitates the use of potentially harmful agents such as organic solvents, chemical initiators, or UV irradiation. Residual chemicals can leach from the hydrogels, whereas UV-based polymerization techniques are incompatible with therapeutic proteins and cells. ${ }^{13}$ Synthetic hydrogels are therefore often plagued by poor biocompatibility and are challenging from a formulation and regulatory perspective. Biomimetic hydrogels (e.g., peptides, peptoids, etc.) and biohybrid hydrogels (e.g., heparin functionalized polyethylene glycol) may represent a promising option that avoids these issues. ${ }^{14}$ However, these

Received: August 29, 2018

Accepted: November 27, 2018

Published: November 27, 2018 
A

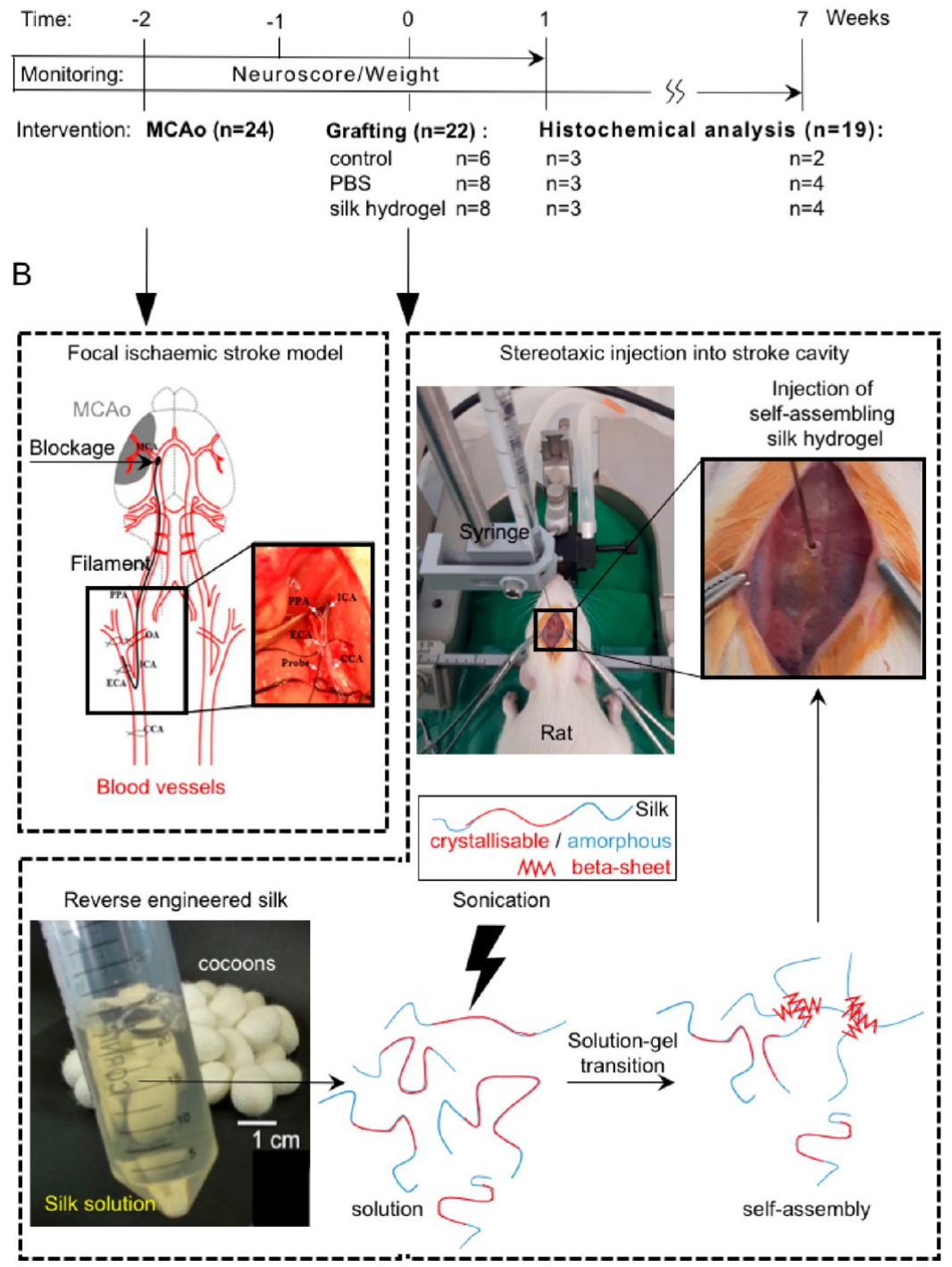

Figure 1. Preparation and administration of self-assembling silk fibroin hydrogels into rats with established focal cerebral ischemic stroke. (A) Experimental timeline, stroke intervention and assessment. Right transient middle cerebral artery occlusion (MCAo) was performed on 24 rats 2 weeks prior to the grafting surgery. At time 0 , animals $(n=22)$ were randomly assigned to either (i) control (no injection, stroke only) ( $n=6)$, (ii) stereotactic PBS injection (vehicle) $(n=8)$ and (iii) stereotactic $4 \% \mathrm{w} / \mathrm{v}$ self-assembling silk hydrogel injection $(n=8)$. Neurological assessment was performed for 2 weeks during post-MCAo recovery and at 1 week postgrafting. Animals were euthanised at 1 week (subacute phase) and 7 weeks (delayed phase) post grafting and subjected to (immuno)-histochemical analysis. (B) Schema of the right transient middle cerebral artery occlusion (MCAo). (MCAo image photograph and diagram reproduced with permission from ref 31. Copyright Neural Regeneration Research, Wolters Kluwer Medknow Publications.) Self-assembling silk fibroin hydrogels were freshly prepared by reverse engineering Bombyx mori silk cocoons. A $4 \% \mathrm{w} / \mathrm{v}$ silk fibroin solution was sonicated (i.e., energy input) first to initiate the solution-gel transition (i.e., self-assembly); during this transition, the stereotactic injection into the stroke cavity was performed.

systems still require significant research efforts to establish selfassembling hydrogels with the necessary mechanical properties and biocompatibility for in vivo administration and subsequent clinical exploitation. A broad range of naturally derived biopolymers has been proposed for use in therapeutic payload delivery. For example, collagen-based hydrogels have been extensively studied, ${ }^{15}$ but these have poor mechanical properties and show limited resistance to biodegradation (or the ability to fine-tune this). Their predominantly bovine origin also raises a theoretical risk of introducing prions directly into the brain, which raises major caveats against collagen-based systems.

One naturally occurring polymer with a robust human safety profile is the silk fibroin from Bombyx mori, which has been approved for human use for decades as a suture material. ${ }^{16}$ The silk fibroin degrades in vivo ${ }^{14,16}$ and silk can be processed under entirely aqueous conditions under ambient conditions ${ }^{17}$ to create a regenerated silk fibroin solution that can be processed into a variety of formats, ${ }^{18,19}$ including self-assembling hydrogels. For use in the stroke setting, these self-assembling hydrogels can be finely tuned to closely match brain mechanics. ${ }^{20}$ In addition, the solution-gel transition of silk fibroin can be controlled enabling minimally invasive administration; a key feature for their proposed use in the stroke setting. Recent progress by Fernández-Garcia and co-workers has demonstrated that the injection of self-assembling silk hydrogels into the caudate putamen (striatum) of healthy mouse 
brains showed no decline in cognitive function or animal behavior over the 6 week study period. ${ }^{21}$ Work by the same group also showed that delivery of mesenchymal stem cells into the stroke lesion using self-assembling silk hydrogels resulted in improved functional recovery and restitution of damaged circuitry. ${ }^{22}$ These seminal studies, ${ }^{21,22}$ in addition to prior work in spinal cord, ${ }^{23}$ cardiac, $^{24}$ ligament, ${ }^{25}$ vasculature, ${ }^{18,26}$ and cancer tissues, ${ }^{27}$ have successfully used silk fibroin biomaterials for tissue engineering and to deliver advanced therapeutics payloads (e.g., proteins, antibodies etc.). ${ }^{14}$ Nonetheless, dedicated biocompatibility studies are needed that examine the performance of silk fibroin hydrogels in the rodent brain after stroke.

In the present study we used stereotactic injection to deliver self-assembling silk fibroin hydrogels $(4 \% \mathrm{w} / \mathrm{v})$ into experimental stroke lesions to establish their capacity for good space conformity in the stroke cavity, their interaction with the glial scar, their provocation of inflammatory responses, and their ability to support endogenous cell proliferation in vivo after experimental stroke.

\section{MATERIALS AND METHODS}

Silk Fibroin Hydrogel Manufacture. From silk cocoons, silk fibroin was extracted and turned into an aqueous solution as described previously. ${ }^{27}$ Briefly, Bombyx mori cocoons were cut into $\sim 25 \mathrm{~mm}^{2}$ pieces, boiled for $60 \mathrm{~min}$ in $\mathrm{Na}_{2} \mathrm{CO}_{3}$, and then rinsed in $\mathrm{ddH}_{2} \mathrm{O}$ to remove sericin proteins. Extracted silk fibroin was subsequently airdried and dissolved in $9.3 \mathrm{M} \mathrm{LiBr}$ at $60{ }^{\circ} \mathrm{C}$. This solution was dialyzed (molecular weight cutoff 3500 ) against $\mathrm{ddH}_{2} \mathrm{O}$ for $48 \mathrm{~h}$ to remove the $\mathrm{LiBr}$ salt. Next, $10 \times$ phosphate buffered saline (PBS) was added to the silk fibroin solution to obtain physiological osmolarity of the final preparation. The resulting $4 \% \mathrm{w} / \mathrm{v}$ silk fibroin solution was filter sterilized. Gelation was induced by sonicating the silk fibroin solution using a Branson Digital Sonifier 450 (Branson Ultrasonics, Danbury, CT, USA) at $15 \%$ amplitude for 15 to $45 \mathrm{~s}$. The sonicated silk fibroin samples were then filled into Hamilton syringes prior to the onset of gelation and injected into the animal before the solution-gel transition was completed. (Figure 1B).

In Vivo Experimental Design. All in vivo studies were approved by the Home Office of the United Kingdom (Project License number 60/4469). All procedures complied with the UK Animals (Scientific) Procedures Act (1986) and the Ethical Review Process of the Institute of Pharmacy and Biomedical Sciences of University of Strathclyde in adherence with ARRIVE guidelines. ${ }^{28}$ First, animals were subjected to the middle cerebral artery occlusion (MCAo) reperfusion stroke model (detailed below). Two weeks after MCAo, the animals were randomly assigned to one of the 3 study groups, namely (i) control (i.e., no injection), (ii) PBS injection, and (iii) $4 \% \mathrm{w} / \mathrm{v}$ self-assembling silk fibroin hydrogel injection. Animals were euthanised at 1 or 7 weeks post-transplantation to probe the acute and delayed response toward the graft (Figure 1A).

Middle Cerebral Artery Occlusion (MCAo). The experimental focal stroke model was the middle cerebral artery occlusion (MCAo) reperfusion model by intraluminal thread, ${ }^{29}$ which leads to progressive cavitation in one hemisphere. ${ }^{5,30}$ The model produces different distributions of lesions, depending on the extent of the ischemia, and has been described elsewhere as striatal only or encompassing both striatal and cortical (or full) lesions. ${ }^{32-34}$ Male Sprague-Dawley rats (weight 240-290g, 8-9 weeks, Harlan, UK, $n=24$ ) were maintained on a $12 \mathrm{~h}$ light/dark cycle with food ad libitum. The right MCA was occluded for $60 \mathrm{~min}$ by insertion of a propylene filament (Doccol Corporation, USA, tip diameter with coating $0.33 \pm 0.02 \mathrm{~mm}$ ), via the common carotid artery to the ostium of the MCA in the circle of Willis, as previously described. ${ }^{35}$ Isoflurane (4\% for induction, $2 \%$ for maintenance in $30 \%$ oxygen) was used for anesthesia during insertion and removal of the filament and the body temperature was maintained at $37 \pm 1{ }^{\circ} \mathrm{C}$. A priori exclusion criteria were any animal found to be moribund due to excessive weight loss (>20\% of start weight) or animals that exhibited no neurological deficit. The severity of the deficit was established by monitoring the animals for the following three week period, animals for neurological deficits using a grading scale of 0 to $4^{36}$ (with modifications where $0=$ no observable deficit; $1=$ forelimb flexion; 2 = decreased resistance to lateral push (and forelimb flexion) without circling; and $3=$ decreased resistance to lateral push (and forelimb flexion) with circling. An additional score $=4$ was added if the animal appeared unstable or exhibited reduced spontaneous motility.

Stereotactic Surgery. Two weeks after MCAo, the rats were anaesthetized with isoflurane (4\% induction, $2 \%$ maintenance) and randomly assigned to receive no graft at all (control) $(n=6)$, PBS $(n=$ $8)$ or $4 \%(\mathrm{w} / \mathrm{v})$ self-assembling silk fibroin hydrogel $(n=8)$. Animals were placed in a stereotactic frame and injections $(10 \mu \mathrm{L}$ at a $2 \mu \mathrm{L} / \mathrm{min})$ were performed at coordinates $(\mathrm{L})-1.5 \mathrm{~mm},(\mathrm{~A}-\mathrm{P})-3.5 \mathrm{~mm}$ and $(\mathrm{V})$ $-6.5 \mathrm{~mm}$, using a $10 \mu \mathrm{L}$ Hamilton syringe with a $22 \mathrm{G}$ blunt tip needle. The experimental approach and timelines for this study are shown in Figure 1.

Histology and Immunofluorescence. Animals were terminally anaesthetised at either 1 week or at 7 weeks after the transplantation by overdosing with sodium pentobarbital $(60 \mathrm{mg} / \mathrm{kg}$ i.p. $)$. A transcardial perfusion of $0.9 \%$ saline was followed by $4 \%$ ice-cold paraformaldehyde in $0.2 \mathrm{M}$ phosphate buffered saline (PBS). Brains were removed following craniotomy and were fixed in paraformaldehyde for $24 \mathrm{~h}$. The brains were then immersed in cryoprotective solution (30\% sucrose in PBS with $0.01 \%$ sodium azide $(\mathrm{NaAz})$ for $72 \mathrm{~h}$, followed by rapid freezing on dry ice. Coronal $(40 \mu \mathrm{m})$ cryostat sections were cut throughout the MCA territory. The experimenter was blinded to the experimental groups by recoding the tissue slides. The tissues were stained with hematoxylin and eosin (H\&E) to visualize the silk fibroin hydrogel graft within the cavity and the lesion topography. Whole brain images were captured (Samsung Galaxy Neo camera, CMOS 16.0 MP resolution, with $\mathrm{f} / 1.9$ aperture) to identify lesion/graft localization in H\&E-stained sections and were used as adjacent sections to represent location of immunostaining as described below.

For immunohistochemistry, sections were incubated in $10 \%$ concentrations of the appropriate blocking sera and in PBS with $0.3 \%$ $\mathrm{v} / \mathrm{v}$ Triton X-100 for $40 \mathrm{~min}$ prior to overnight incubation with primary antibodies (diluted in PBS with $10 \% \mathrm{v} / \mathrm{v}$ normal serum and $0.3 \% \mathrm{v} / \mathrm{v}$ Triton X-100) at $4{ }^{\circ} \mathrm{C}$. Primary antibodies consisted of rabbit antiGFAP (1:1000, Z0334, DAKO, USA, CA) to visualize the glial scar and to quantify the volume of the cavity, rat anti-CD11b (1:200, ab1211, Abcam, UK) to detect microglia/macrophages, rabbit anti-Ki67 (1:500, ab15580, Abcam, UK) to detect proliferating cells and mouse antiRECA-1 to detect vascular endothelial cells (1:100, ab9774, Abcam, UK). After incubation with primary antibodies, sections were washed three times for $5 \mathrm{~min}$ in PBS and then incubated with a secondary antibody for $2 \mathrm{~h}$ at room temperature. Secondary antibodies consisted of appropriate fluorescent Alexa 488 or Alexa 555 antibodies (1:500, Invitrogen, UK). Sections were rinsed three times for $5 \mathrm{~min}$ in PBS before application of Vectashield with DAPI (Vector Laboratories, UK). Images were captured and analyzed using WinFluor V3.9.1 (Nikon Eclipse E600). GFAP positive staining labels the glial scar, showing the bordered outline of the cavity. Therefore, GFAP was used to quantify lesion volume. The border outlined by GFAP was transcribed onto line diagrams and the areas measured by ImageJ and the volume calculated over the number and thickness of sections that exhibited tissue loss.

Statistical Analyses. Animal weight and neurological deficit data were shown as individual animal data points and also expressed with mean \pm standard error of mean (S.E.M.) and analyzed by one-way ANOVA with a Bonferroni posthoc test. Correlations of number of Ki67+ cells versus cavity volume were analyzed by regression analysis (Prism 6; GraphPad Software Inc., USA, CA). A $P$ value of $<0.05$ was considered significant.

\section{RESULTS}

Impact of Silk Fibroin Hydrogels on Morbidity or Mortality. Given the potential adverse effects of cerebral 
implants, we monitored neurological deficit and weight loss before and after grafting (Figure 2A, B). Neurological deficit
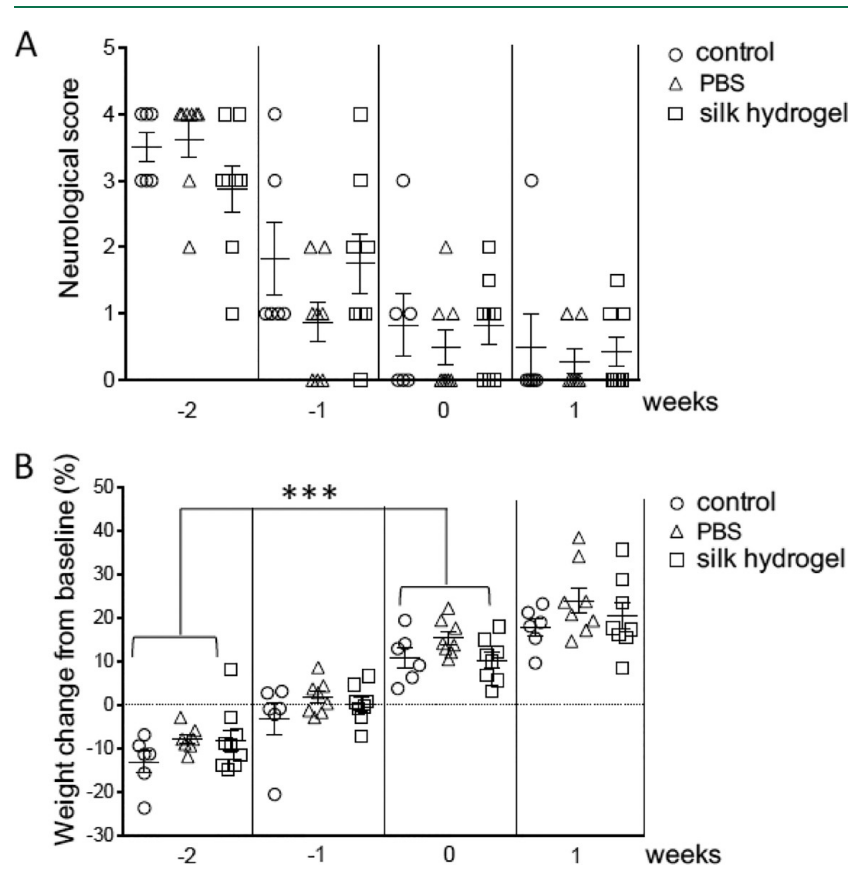

Figure 2. Neurological function and body weight were unaffected by silk fibroin hydrogel implants. (A) General neurological deficits and (B) body weight changes in control $(n=5)$, PBS $(n=7)$, and silk fibroin hydrogel implanted rats $(n=7)$ at $24 \mathrm{~h}($ week -2$)$ and 7 days (week -1 ) post-MCAO and pregrafting (week 0 ) and 7 days postgraft (week 1) (one way ANOVA, Bonferrroni $t$ test, $* * * P<0.001$ ).

scores were highest $24 \mathrm{~h}$ after MCAo (week -2; Figure 2A). During the post-MCAo recovery period, prior to grafting, 4 rats had full restoration of their neurological functions with no observable deficit (score $=0$ ) (those rats with minor or striatal only stroke), whereas 18 showed only partial recovery (those with a full cortical stroke) (Figure 2A). Regression analysis confirmed this positive correlation between neurological score and cavity volume in each group $(P<0.05)$ (Figure $\mathrm{S} 1)$.

Any contribution of silk fibroin hydrogel grafting to neurologic deficit was assessed at 1 week postgrafting. No significant differences were observed in the silk fibroin hydrogel grafted animals either compared to before grafting or compared to control or PBS treated animals (Figure 2A). Regression analysis confirmed no effect of silk fibroin hydrogel administration on the correlation of neurological score (measured at $24 \mathrm{~h}$ postgrafting) with cavity volume (measured at 1 and 7 weeks postgrafting) as the difference between slopes was not significant $(\mathrm{F}(2,13)=1.25, P=0.3)$ (Figure $\mathrm{S} 1)$.

The rats typically had a weight drop $24 \mathrm{~h}$ after MCAo (week -2 ; Figure $2 \mathrm{~B}$ ), and by the end of the 2 week recovery period, all animals had gained more than their pre-MCAo weight $(\mathrm{F}(5,38)$ $=37.89, P<0.0001$, one way ANOVA). No significant differences were observed in body weight at 1 week postgrafting in the silk fibroin hydrogel grafted animals when compared to control or PBS rats $(\mathrm{F}(1.983-13.88)=1.3, P=0.3$, one way ANOVA). No animals were excluded from any group due to excessive weight loss, based on the above-mentioned exclusion criteria. However, one animal experienced $>20 \%$ weight loss but was spontaneously mobile and was eating and drinking well and therefore did not fall within the exclusion criteria. One premature death occurred during MCAo surgery that was attributable to anesthetic accident and another death at $6 \mathrm{~h}$ postreperfusion was due to subacute respiratory distress. One rat died at the 7 weeks time point in the control group, attributed to severe brain edema due to the stroke after ruling out hemorrhagic transformation and on observation of an enlarged ipsilateral hemisphere. The included animals are detailed in Figure 1A. Data from two other rats were excluded (silk fibroin hydrogel $(n=1)$ and PBS $(n=1)$ both at 1 week postgraft due to damage to the tissue during histological processing.

Space Conformity of Self-Assembling Silk Fibroin Hydrogels over Time. The MCAo treatment resulted in two main infarct populations across the three treatment groups at both time points. Cortical-striatal lesions exhibited extensive tissue loss, resulting in a "large cavity" (Figure 3B, D, left panel, black box) and further magnification showed that all the
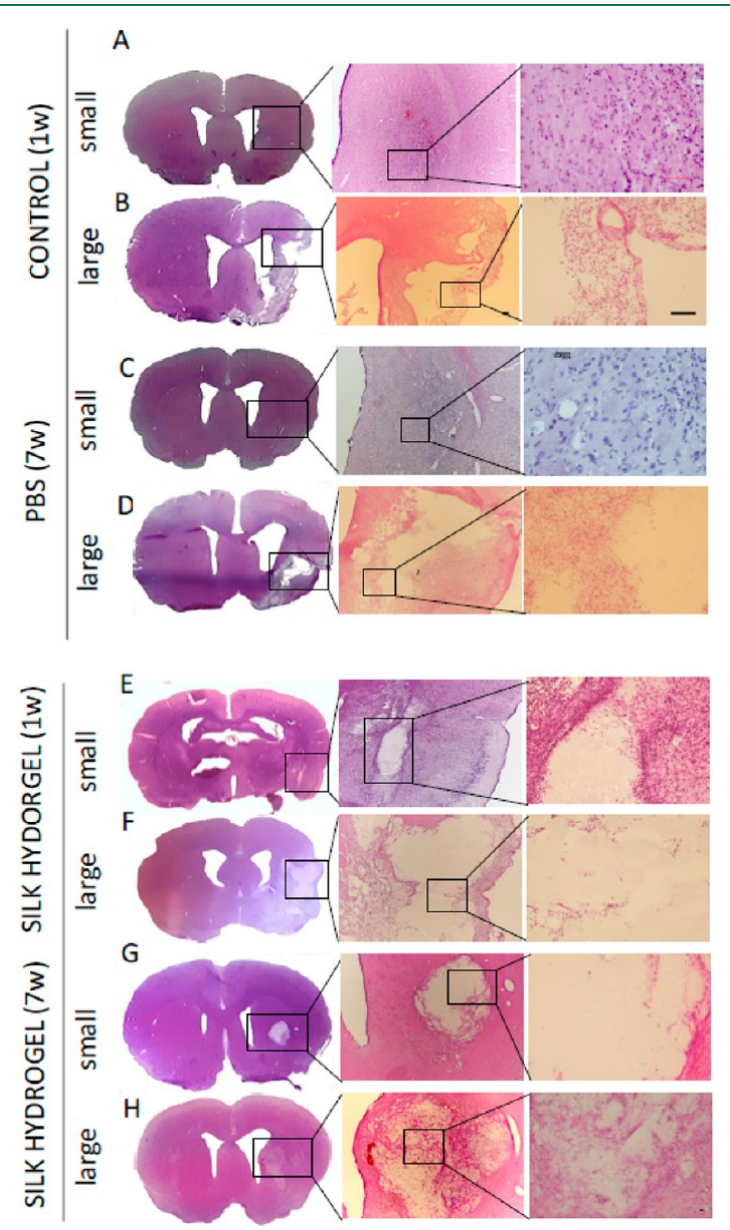

Figure 3. Self-assembling silk fibroin hydrogels exhibited good space conformity and retention in small and large stroke cavities. (A-D, F$\mathrm{H})$ Representative hematoxylin and eosin stained coronal sections at the level of the nucleus accumbens (IA $10.56 \mathrm{~mm}$ ) and (E) the level of the anterior hypothalamus (IA $6.84 \mathrm{~mm}$ ) transplanted either with (E$\mathrm{H})$ self-assembling silk fibroin hydrogels, (C, D) PBS only, or (A, B) with no-injection (control) at (A, B, E, F) 1 or $(\mathrm{C}, \mathrm{D}, \mathrm{G}, \mathrm{H}) 7$ weeks. The whole brain sections and magnified figures illustrate the presence and structure of the silk fibroin hydrogel graft in the stroke lesion (visualized by light pink eosin staining), surrounded by the dead tissue (pyknotic nuclei stained with hematoxylin (purple) in both small (E and $\mathrm{G})$ and large $(\mathrm{F}$ and $\mathrm{H})$ cavities or the presence of an empty stroke cavity in (A, B) control or (C, D) PBS-treated animals. Please see Figure S2 for higher magnification of representative hematoxylin and eosin-stained sections at the regions of interest. Scale bars: $100 \mu \mathrm{m}$. 

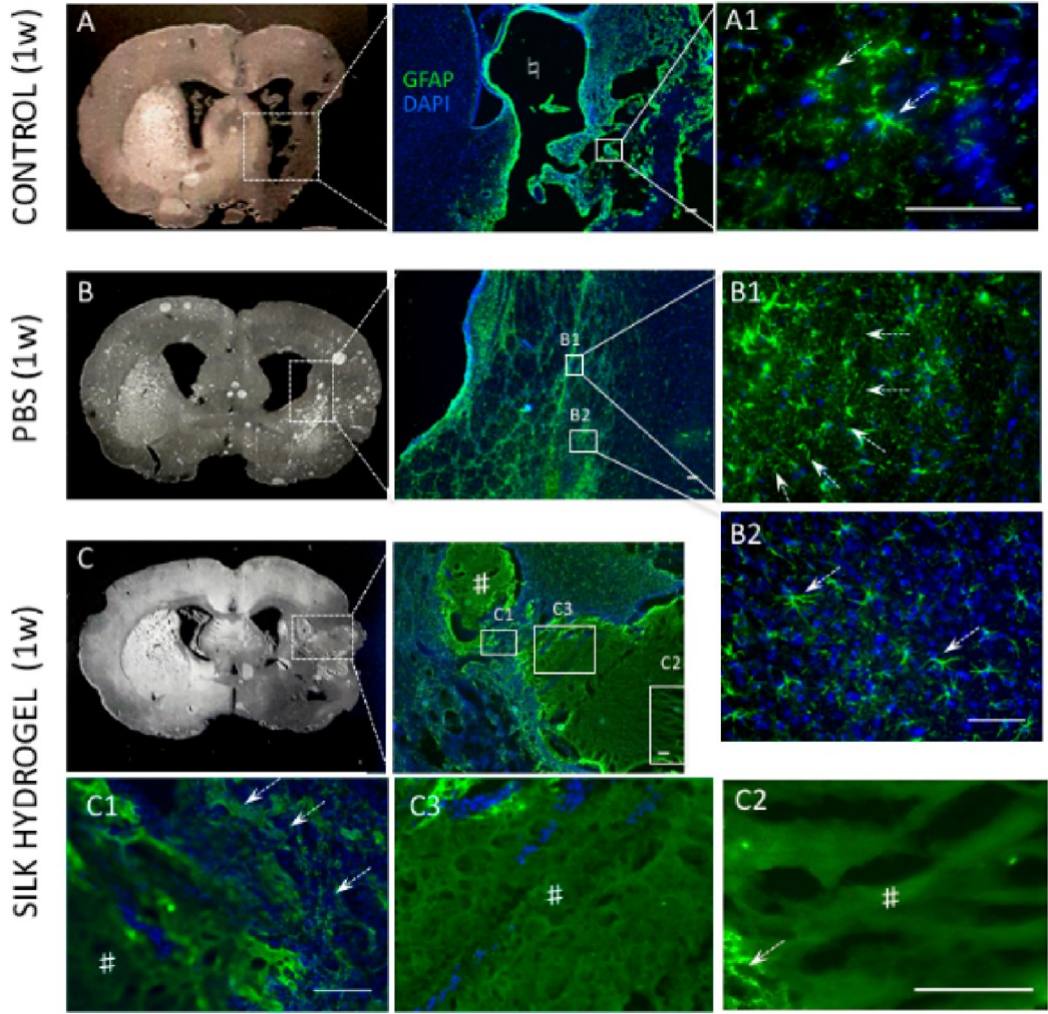

Figure 4. Silk fibroin hydrogels interfaced closely with the glial scar. Activated astrocytes (green $\mathrm{GFAP}^{+}$cells; dotted white box and dotted arrows; DAPI nuclei in blue), staining at coronal levels of the (A, B) nucleus accumbens (IA $10.56 \mathrm{~mm}$,) and (C) globus pallidus (IA $7.68 \mathrm{~mm}$ ) illustrating the location of the cortical or striatal voids. (A) Stereotaxic sham control and (B) PBS animals (clear whole brain section which is adjacent section to that immunostained) surrounded by activated astrocytes or (C) silk fibroin hydrogel-treated animals 1 week post grafting; the cavity is filled with silk hydrogel (green color, hash symbols in $\mathrm{C}$ and $\mathrm{C} 1-\mathrm{C} 3$ ) and (C1, arrows) a silk fibroin hydrogel-glial scar interface is visible. Scale bars: $100 \mu \mathrm{m}$.

remaining cells, as indicated by pyknotic nuclei (dark purple) revealed by hematoxylin staining (right panel; black box). By contrast, the striatal-only lesions exhibited a "small cavity" (Figure 3A, C). The silk fibroin hydrogel grafts from a total analysis of 7 brains (>250 sections per brain) were always localized in the lesion cavity. In animals with either small or large stroke cavities, the self-assembling silk fibroin hydrogel filled the lesion and was evenly spread throughout the entire stroke cavity at 1 week (Figures 3E, F). The silk fibroin hydrogel was recognized by its light pink color (black box, Figure 3E, F). In a similar manner, the silk fibroin hydrogel graft showed good space conformity at 7 week time point, for both small (Figure $3 \mathrm{G}$ ) and large (Figure 3H) cavities, with a good signs of retention and no visible signs of hydrogel degradation. The selfassembling silk fibroin hydrogels could also be readily visualized by immunofluorescent dyes due to dye adsorbance, as described below.

Impact of Silk Fibroin Hydrogels on the Adjacent Glial Scar. A tight host tissue-hydrogel interface within the stroke cavity is required to provide a good support matrix for cell infiltration/proliferation and for good delivery of therapeutic payloads in order to promote repair processes. We therefore assessed coronal sections at, and adjacent to, the cavity for $\mathrm{GFAP}^{+}$cells, a marker of reactive astrogliosis, the predominant constituent of the glial scar and of an inflammatory response (Figure 4). The ischemic cavity was surrounded by a glial scar, consisting of activated astrocytes (GFAP+, enlarged soma with more and thicker processes, dotted arrows, Figure 4A1, B1, B2). The self-assembling silk fibroin hydrogel filled the cavity (green staining, hash sign) Figure 4C), and was surrounded by the remaining glial scar (dotted arrows, Figure 4C1). A subtle change in the structure of the silk fibroin hydrogel was observed throughout the cavity, with a less dense composition in the epicenter (Figure 4C2) and a "bubbly" structure in the tissue adjacent to the epicenter (Figure 4C3). The silk fibroin hydrogel was interspersed in the surrounding glial scar (Figure 4C1), indicating an interaction between the silk fibroin hydrogel and the glial scar. No colocalization of DAPI staining with the $\mathrm{GFAP}^{+}$staining was observed in any of the sections studies (for example see Figure 4C1).

Impact of Silk Fibroin Hydrogels on Microglial/ Macrophages in the Stroke Cavity. The effects of silk fibroin hydrogel on inflammation and the glial scar were examined by double labeling of $\mathrm{CD} 1 \mathrm{~b}^{+}$(microglia/macrophages) and $\mathrm{GFAP}^{+}$staining. In control and PBS-injected animals (Figure 5A, B), at the 1 week time point, $\mathrm{CD} 11 \mathrm{~b}^{+}$cells (arrows) were observed within the lesion core and were often associated with activated astrocytes (enlarged soma with many and thick processes), as part of the glial scar (dotted arrows) in both cortical (control, Figure 5A2) and striatal lesions (PBS, Figure 5B2). Tissue at the coronal level of the globus pallidus (Figure 5C) and the coronal level of the nucleus accumbens (Figure 5D) were studied as representatives of the posterior and anterior extents of the lesion, respectively. Unlike in the control and PBS-treated animals, animals with silk hydrogel grafts had no evident microglia/macrophages in the core of the lesion; instead, the core of the lesion was filled with a silk graft (Figure $5 \mathrm{C} 1$, hash symbol) and surrounded by the glial scar (dotted outline), which had associated microglia/macrophages (red outline) (Figure 5C2, merged and single color figures). A 

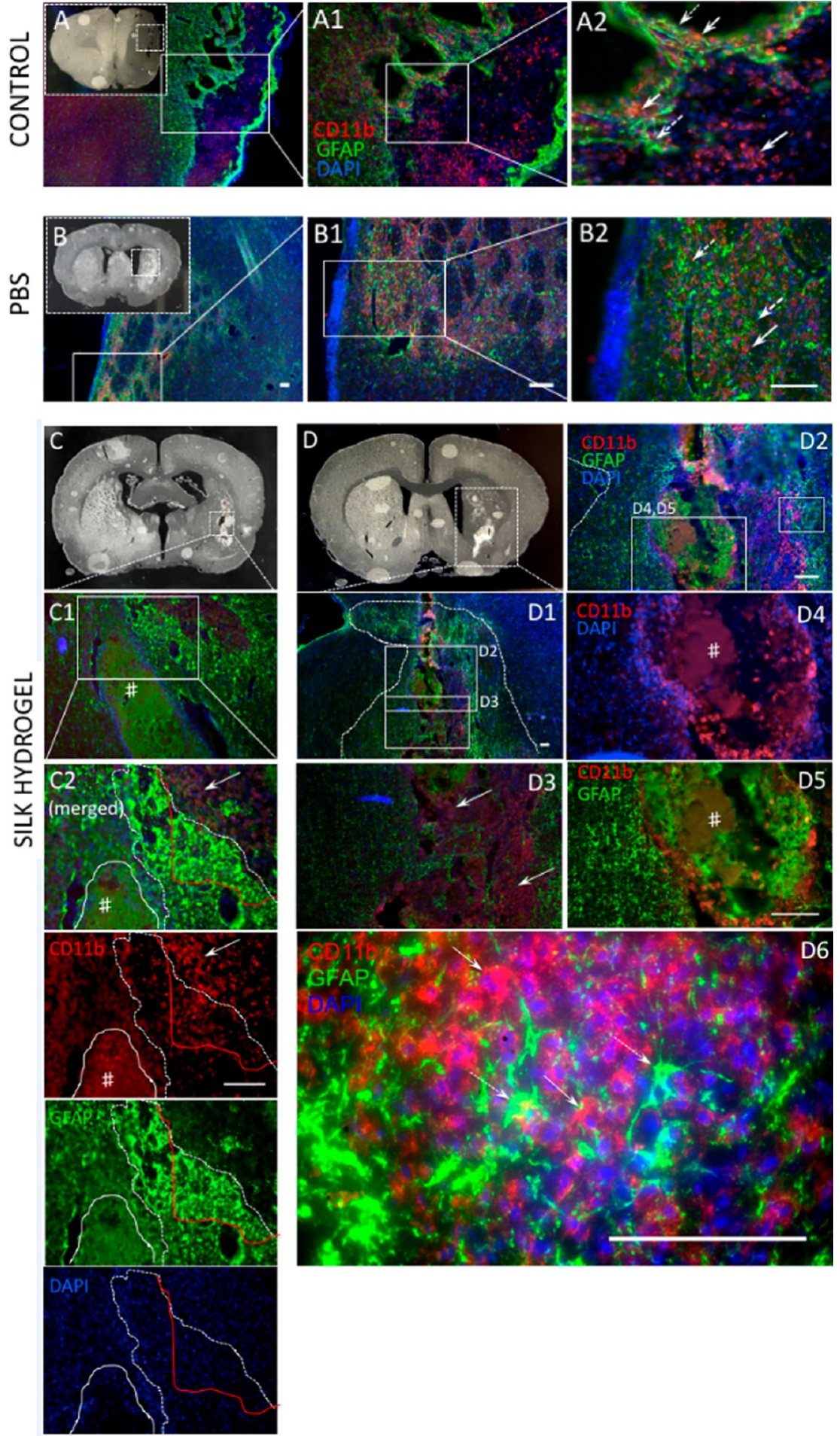

Figure 5. No increase in microglial/macrophage response toward the self-assembling silk fibroin hydrogel in the stroke cavity at week $1 . \mathrm{CD}_{11} \mathrm{~b}^{+}($red $) /$ $\mathrm{GFAP}^{+}$(green)/DAPI(nuclei in blue) staining at coronal levels of (A) no-injection control at the level of the olfactory tract (IA $12.72 \mathrm{~mm}$ ) and (B) PBS injection at the level of the nucleus accumbens (IA $10.56 \mathrm{~mm}$ ) illustrating location of microglial activation in relation to the stroke lesion and glial scar. CD $11 b^{+}$cells were activated (amoeboid shape with large soma, arrows) within the lesion core and often associated with activated astrocytes as part of the glial scar (dotted arrows in higher magnification, right-hand panels). (C, D) Self-assembling silk fibroin hydrogels (green, hash symbol with white outline) in the stroke cavity lack CD11+ cells in both (C1) posterior (globus pallidus) and (D) anterior (nucleus accumbens) small lesion sites. $\mathrm{CD} 11^{+}$cells were observed some distance away from the silk fibroin hydrogel graft (C1, arrow and red line; D1 and D4, asterisk). The silk fibroin hydrogel was surrounded by the glial scar (C2 and D2, dotted white outline) containing microglia/macrophages (D5-D7 arrows = microglia/ macrophage and dotted arrows $=\mathrm{GFAP}^{+}$astrocytes). Scale bars: $100 \mu \mathrm{m}$.

microglia/macrophage agglomerate (Figure 5C2, arrow and red outline) was observed at some distance from the silk hydrogel. In the more anterior part of the lesion, again no $\mathrm{CD} 11 \mathrm{~b}^{+}$cells were evident inside the silk hydrogel (Figure 5D2, D4, D5, hash symbol), but a substantial number of microglia/macrophages cells were located next to it and toward the edge of the lesion 

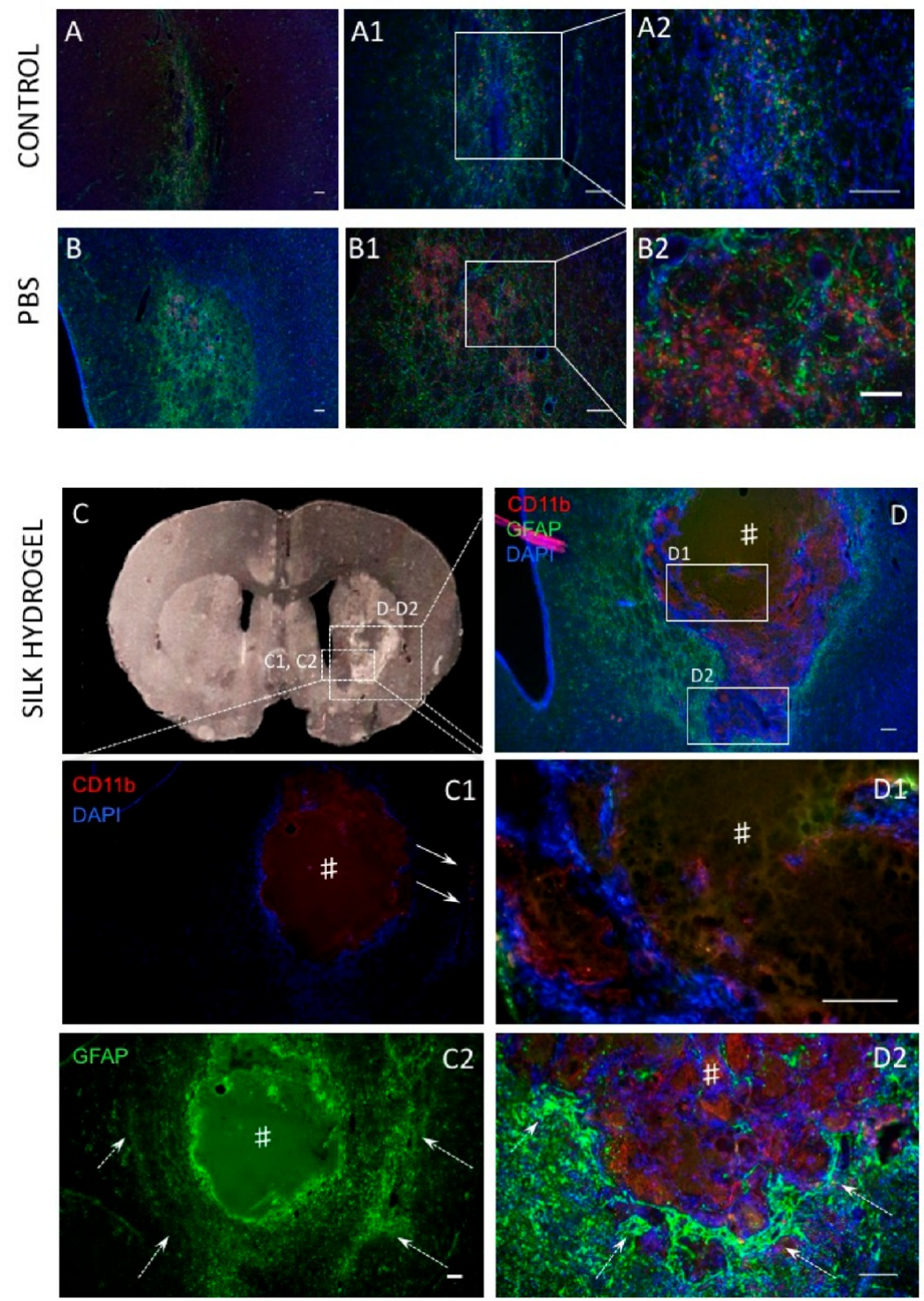

Figure 6. No increase in the microglial/macrophage response toward the self-assembling silk fibroin hydrogel in the stroke cavity at week $7 . \mathrm{CD} 1 \mathrm{~b}^{+}$ (red)/GFAP ${ }^{+}$(green)/DAPI (nuclei in blue) staining at the coronal level of the nucleus accumbens with a lack of CD11 $1^{+}$cells in the lesion (B1) and the whole silk graft (hash symbol) surrounded by the glial scar (B2, arrows, C1 dotted arrows). Microglia/macrophages were observed at the edge of glial scar, remote from the silk fibroin hydrogel (C, arrows; B2, dotted arrows). Scale bars: $100 \mu \mathrm{m}$.

(Figure 5D1, dotted line, D3, solid arrows) and at the border region (Figure 5D6, solid arrows), in close interaction with GFAP+ astrocytes (Figure 5D6, dotted arrows).

A similar response was observed at 7 weeks, where in control and PBS there were no or very little CD11b+ microglia/ macrophages visible in the lesion area (Figure 6A, B). After injection, where the striatal lesion was filled with a silk fibroin hydrogel graft (hash symbol) (Figure 6C, D), no microglia/ macrophages were evident in the silk fibroin hydrogel graft (Figure 6D, D1) and the whole silk fibroin graft was surrounded by the glial scar (Figure 6D2, dotted arrows). At a more rostral coronal level (Figure 6C), microglia/macrophages were again observed at the edge of lesion (Figure 6C1, solid arrows) but $>300 \mu \mathrm{m}$ from the silk fibroin hydrogel graft (hash symbols) and along the glial scar border (Figure 6C2, dotted arrows).

Impact of Silk Fibroin Hydrogels on Endogenous Cells in the Lesion Cavity. Extensive nuclear staining (DAPI positive), as shown in Figures 4-6, that did not colocalize with GFAP or CD11b was observed in the silk fibroin hydrogel animals. For this reason, we investigated whether silk fibroin hydrogels might promote cell proliferation. After MCAo, we observed many RECA- $1^{+}$cells, indicating considerable angiogenesis, but we observed only a low number of proliferating cells (teal = green+blue, Figure $7 \mathrm{~A})$, which were surrounded by RECA- $1^{+}$cells in control animals (Figure 7A1). However, animals grafted with the self-assembling silk fibroin hydrogel showed many $\mathrm{Ki} 7^{+}$cells, indicating the presence of proliferating cells in the cortical area (Figure 7B) that were interacting with areas of angiogenesis (RECA- $1^{+}$) and some unidentified cell type $\left(\mathrm{DAPI}^{+}\right)$, resulting in an intimate heterotypic cell cluster (Figure 7B2). No such structures were found in control animals (Figure 7A1). The RECA- $1^{+} / \mathrm{Ki}^{+} 7^{+} /$ $\mathrm{DAPI}^{+}$cells (arrows) were located at the edges of the silk graft (Figure 7C, C1, hash symbol).

The relationship between the volume of the lesion and the number of proliferating cells was also investigated. The number of proliferating was significantly influenced by lesion volume and silk fibroin hydrogel treatment (Figure 7D). The control animals 

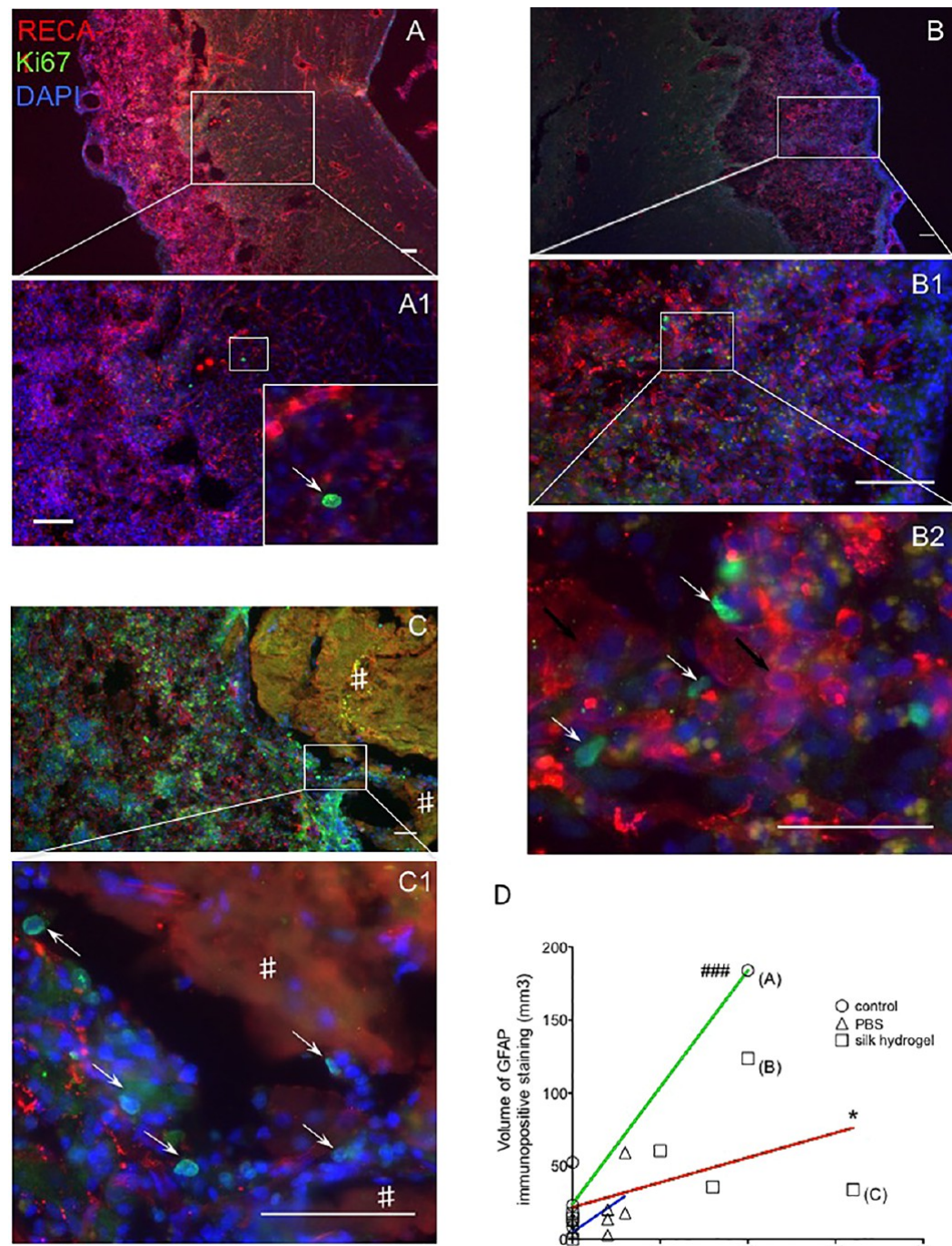

D

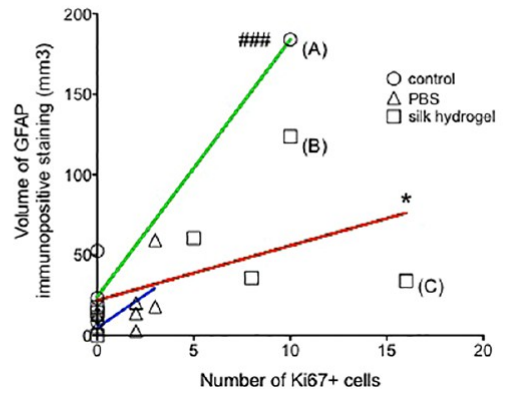

Figure 7. Silk fibroin hydrogels supported endogenous cell remodelling of the stroke lesion. RECA- $1^{+}$(red)/ Ki67 $7^{+}$(green)/ DAPI (nuclei in blue) staining in (A) representative control with substantial RECA- $1^{+}$cell staining (red) but a low number of Ki67 $7^{+}$cells $($teal $=$green + blue $)(A 1$, white arrow for a Ki67+ single cell). (B) Silk fibroin hydrogel implanted animals showed many Ki67 $7^{+}$cells present in the cortical area (B1), interacting with new vessels $\left(\right.$ RECA- $\left.1^{+}\right)$(B2, arrows). RECA- $1^{+}$vessels (black arrow), host proliferating cells (white arrows), and some other type of cell (DAPI), existing as one conglomerate (B2) RECA $-1^{+} / \mathrm{Ki}^{+} 7^{+} / \mathrm{DAPI}^{+}$cells were located inside the silk fibroin hydrogel graft (sharp symbol, C and C1 (white arrows Ki6 $7^{+}$cells). (D) Relationship (line of best fit) between the number of Ki67 $7^{+}$in the stroke cavity and lesion volume (expressed as volume of GFAP+ immunostaining). Control slope (green line): gradient $r^{2}=0.94$ (95\% CI: 0.96, 1.0), ${ }^{\# \# ~} P<0.001$. Silk fibroin hydrogel slope (red line): gradient $r^{2}=0.25(-0.7,0.86), P=0.78$. Difference between slopes $* P<0.05$. Scale bars: $100 \mu \mathrm{m}$.

showed a significant positive correlation between the lesion size and the number of proliferating cells $\left(r^{2}=0.94, P<0.01\right)$. Posttreatment with the silk fibroin hydrogel abrogated this positive relationship $\left(r^{2}=0.24\right)$ and significantly decreased the gradient of the relationship compared to control (difference between gradients $\mathrm{F}(2,13)=5.94, P<0.05)$.

\section{DISCUSSION}

The key findings in the present study were that self-assembling silk hydrogels do not impact on morbidity; exhibit good space conformity and retention in the stroke cavity; interact with the glial scar; provoke no overt inflammatory response; and support endogenous cell proliferation in vivo after experimental stroke.

Given the adverse effects associated with some biomaterials in vivo, ${ }^{11,37,38}$ we first tested for any potential overt behavioral effects following implantation of $4 \% \mathrm{w} / \mathrm{v}$ silk fibroin hydrogels into the stroke cavity; this selected silk fibroin concentration results in hydrogels with similar mechanical properties of healthy brain tissue (Figure S3). ${ }^{20}$ At 2 weeks post-MCAo, the silk fibroin hydrogel graft had no significant effect on body weight or neurological score when compared to the no graft control or PBS, implying no overt adverse effects of intracranial silk fibroin hydrogels. These results are in line with a previous study in healthy C57BL/6 mice, in which $5 \mu \mathrm{L}$ of $2 \% \mathrm{w} / \mathrm{v}$ silk fibroin hydrogel was injected into the caudate putamen. These mice showed no cognitive or sensorimotor deficits according to a set of behavior and electrophysiological assessments. ${ }^{21} \mathrm{We}$ speculate that the absence of silk fibroin hydrogel swelling, the minimally invasive application route, the absence of any chemical cross-linker and the good biocompatibility typically observed with silk fibroin contributed to the excellent tolerability of self-assembling silk fibroin hydrogels in the stroke brain. ${ }^{39}$ Overall, these findings indicate that the silk fibroin hydrogel grafting had no effect on mortality, body weight or 
neurological behavior after MCAo in animals with large or small cavities. Therefore, this study allowed assessment of silk hydrogel effects on space conformity and host tissue architecture in large and small cavities at both subacute (1 week) and delayed (7 weeks) time points.

As is the case in stroke patients, where a large heterogeneity in outcome exists, variability in the lesion within a group of animals exposed to MCAo with reperfusion by intraluminal thread can be partly attributed to the variable net of sub-branches of the MCA especially observed in Sprague-Dawley rats. ${ }^{40}$ The ischemic damage in the present study was striatal only, extending to the thalamic area, or was a full striatal-cortical lesion; this variability has been previously described after ILT in SD rats. ${ }^{41}$ This allowed assessment of silk fibroin hydrogels in terms of space conformity and host tissue response in both large and small cavities.

In all the animals tested with small and large stroke cavities, the self-assembling silk fibroin hydrogel filled the lesion (bearing in mind the limit of the $10 \mu \mathrm{L}$ injection volume) and was evenly spread throughout the entire stroke cavity implying good space conformity and retention. The silk fibroin hydrogel was interspersed between the tissues having different levels of damage, architecture and density, which would be beneficial for drug and (stem) cell delivery, especially where a cavity is present, ${ }^{6,10}$ as previously shown in spinal cord ${ }^{23}$ and others CNS injuries. ${ }^{10}$

In the present study, the silk fibroin hydrogel was able to modulate glial scar formation. The space taken by the GFAPpositive astrocytes (glial scar) was partially displaced by the silk fibroin hydrogel, potentially decreasing its volume. This type of biomaterial-glial scar interaction has been observed previously. ${ }^{42,43}$ The glial scar formation in the ischemic brain is essential for beneficial effects such as blood-brain barrier repair, limiting the inflammatory response and restricting the ischemic cascade of tissue damage, ${ }^{7}$ but it also has detrimental effects such as precluding axonal regeneration into the damaged tissue ${ }^{44-46}$ and reducing efficacy after cell transplantation. Further studies are required to establish if the interface between self-assembling silk fibroin hydrogels and the glial scar facilitate and/or inhibit these effects on neural repair. Furthermore, how the presence of the silk fibroin hydrogel impacts the (active) remodelling of the glial scar remains an open question.

We did not attempt to fully quantify silk fibroin hydrogel degradation. However, the histological assessment indicated that silk fibroin hydrogels showed no overt signs of degradation in the stroke cavity at either 1 or 7 weeks. This observation contrasts with observations made in healthy mice that had received a $5 \mu \mathrm{L}$ injection of $2 \% \mathrm{w} / \mathrm{v}$ silk fibroin hydrogel into the caudate putamen, where histological assessment revealed an approximately $50 \%$ reduction in hydrogel volume at 4 weeks. ${ }^{21}$ We can only speculate why different observations were made in the present study. For example, the observed volume reduction in the mouse study could simply reflect a collapse of the weaker silk fibroin hydrogel structure in response to tissue stress (which is likely to be absent in the stroke cavity) or a greater resistance of our $4 \% \mathrm{w} / \mathrm{v}$ silk fibroin hydrogels to proteolytic degradation (i.e., a greater total amount of silk fibroin administered). The presence of the silk fibroin hydrogels in the stroke cavity beyond 7 weeks indicates the possibility of supporting tissue regeneration (and/or extended payload release).

One critical aspect is whether the self-assembling silk fibroin hydrogel implants induced an inflammatory reaction over time. We only observed microglia/macrophages within the lesion core at 3 and 9 weeks post-MCAo (the 1 week and 7 weeks postimplant time points) in nongrafted or PBS animals, but no microglia/macrophages were evident in the core of the lesion filled with silk fibroin graft at either 1 or 7 weeks postimplantation (i.e., no colocalization of $\mathrm{DAPI}^{+}$with the $\mathrm{GFAP}^{+}$ signal). The self-assembling silk fibroin hydrogel appeared to fill the cavity, displacing and partially superseding the glial scar and inflamed tissue. Thus, these findings indicate that primary inflammation was caused by the ischemic insult at early timeframes (up to 1 week), as reported previously. ${ }^{7,47,48}$ The silk fibroin hydrogel graft caused no secondary (or additional) inflammation, in agreement with the general observation that silk fibroin hydrogels are typically well tolerated in vivo. ${ }^{14}$

Ischemeic injury is known to stimulate the production of newly proliferated cells. ${ }^{49,50}$ We examined whether selfassembling silk fibroin hydrogels aid endogenous cell proliferation in the ischemic brain by staining for $\mathrm{RECA}^{+}$and $\mathrm{Ki} 67^{+}$ cells, as indicators of angiogenesis and endogenous cell proliferation, respectively. Animals treated with silk fibroin hydrogel showed numerous proliferating cells, which were localized alongside new vessels, in addition to another (unidentified) $\mathrm{DAPI}^{+}$cell type; these heterotopic multicellular structures were only observed in the silk fibroin hydrogel group (Figure 7B2). We further examined the possible modulation of endogenous processes induced in the stroke cavity by the silk fibroin hydrogel by examining the relationship between the lesion volumes and the number of proliferating cells in the stroke cavity in the control and the silk fibroin hydrogel groups and found that the slope of the 2 lines was significantly different (although it should be noted that the extreme point in the control group will have influenced the result), in that silk fibroin hydrogel significantly reduced the gradient compared to control. In any animals with a lesion size at the lower end of the range (e.g., $\sim 50 \mathrm{~mm}^{3}$ ), silk fibroin hydrogel significantly increased the number of proliferating, indicating that smaller lesions are required to attract proliferating cells to the cavity when silk fibroin hydrogel is injected. This is in agreement with many previous in vivo studies which examine hydrogels and neurogenesis ${ }^{51}$ and therefore one might speculate selfassembling silk fibroin hydrogels promote an environment conducive to cell proliferation/infiltration after an ischemic insult. However, further experiments, which are beyond the scope of the present study, are needed to establish the phenotype of these proliferating cells before conclusions can be drawn about the regenerative capacity of self-assembling silk fibroin hydrogels.

\section{CONCLUSIONS}

The present study indicates that self-assembling silk fibroin hydrogels performed well in the ischemic brain of stroked rats. During the solution-gel transition, silk fibroin could be injected into established stroke cavities of variable sizes using a minimally invasive stereotaxic procedure. Furthermore, the silk fibroin hydrogel demonstrated (i) stability over the course of the study (>7 weeks), (ii) excellent space conformity, (iii) good biocompatibility in terms of animal welfare and inflammatory response. We further present preliminary evidence that the silk fibroin hydrogel was able to support endogenous cellular mechanisms. In summary, our findings bode well for the use of this system as a matrix for therapeutic delivery. 


\section{ASSOCIATED CONTENT}

\section{S Supporting Information}

The Supporting Information is available free of charge on the ACS Publications website at DOI: 10.1021/acsbiomaterials. 8 b01024.

Figure S1, neurological score at $24 \mathrm{~h}$ postgrafting in all groups and regression analysis of neurological score versus cavity volume; Figure S2, space conformity and retention of self-assembling silk fibroin hydrogels in stroke cavities; Figure S3, comparison of silk hydrogel with healthy brain tissue and physical performance of silk hydrogels and healthy brain exposed to constant weight loading (9.5 g); all data created during this research are openly available from the University of Strathclyde-Pure, UK Data Service at DOI:10.15129/69e4bc63-c9af-431c82e9-b19e588b0c93 (PDF)

\section{AUTHOR INFORMATION}

\section{Corresponding Authors}

*Email: hilary.carswell@strath.ac.uk.

*Email: philipp.seib@strath.ac.uk.

\section{ORCID}

F. Philipp Seib: 0000-0002-1955-1975

Hilary V.O. Carswell: 0000-0002-0938-1212

\section{Author Contributions}

${ }^{\S}$ H.V.O.C. and F.P.S. contributed equally to this work. N.G. performed the experiments and analyzed and interpreted the data and wrote the manuscript draft. I.O. provided expertise in the manufacture of the silk hydrogels. H.V.O.C. and F.P.S. conceived and designed the research study, interpreted the data, and edited the draft.

\section{Notes}

The authors declare no competing financial interest.

\section{ACKNOWLEDGMENTS}

This research was supported by a Daphne Jackson Fellowship (N.G.) that was sponsored by Medical Research Scotland, an EPSRC First Grant (EP/N03127X/1) (F.P.S.), and a PhD stipend (I.O.) from Taif University, Saudi Arabia. The authors thank Anne Goudie (University of Strathclyde) for technical assistance.

\section{REFERENCES}

(1) Biernaskie, J.; Chernenko, G.; Corbett, D. Efficacy of rehabilitative experience declines with time after focal ischemic brain injury. $J$. Neurosci. 2004, 24, 1245-1254.

(2) Bible, E.; Qutachi, O.; Chau, D. Y.; Alexander, M. R.; Shakesheff, K. M.; Modo, M. Neo-vascularization of the stroke cavity by implantation of human neural stem cells on VEGF-releasing PLGA microparticles. Biomaterials 2012, 33, 7435-7446.

(3) Emerich, D. F.; Silva, E.; Ali, O.; Mooney, D.; Bell, W.; Jin Yu, S.; Kaneko, Y.; Borlongan, C. Injectable VEGF hydrogels produce near complete neurological and anatomical protection following cerebral ischemia in rats. Cell Transplantation 2010, 19 (9), 1063-1071.

(4) Nih, L. R.; Carmichael, S. T.; Segura, T. Hydrogels for brain repair after stroke: an emerging treatment option. Curr. Opin. Biotechnol. 2016, 40, 155-163.

(5) Chesselet, M. F.; Carmichael, S. T. Animal models of neurological disorders. Neurotherapeutics 2012, 9 (2), 241-4.

(6) Massensini, A. R.; Ghuman, H.; Saldin, L. T.; Medberry, C. J.; Keane, T. J.; Nicholls, F. J.; Velankar, S. S.; Badylak, S. F.; Modo, M. Concentration-dependent rheological properties of ECM hydrogel for intracerebral delivery to a stroke cavity. Acta Biomater. 2015, 27, 116130.

(7) Doyle, K. P.; Simon, R. P.; Stenzel-Poor, M. P. Mechanisms of ischemic brain damage. Neuropharmacology 2008, 55 (3), 310-308.

(8) Hardy, J. G.; Scheibel, T. R. Silk-inspired polymers and proteins. Biochem. Soc. Trans. 2009, 37, 677-81.

(9) Holland, C.; Terry, A.; Porter, D.; Vollrath, F. Natural and unnatural silks. Polymer 2007, 48, 3388-3392.

(10) Meng, F.; Modo, M.; Badylak, S. F. Biologic scaffold for CNS repair. Regener. Med. 2014, 9 (3), 367-383.

(11) Nicholls, F.; Gorenkova, N.; Bible, E.; Franco, C.; Chau, D.; Tolias, C.; West, J.; Modo, M. Improving the treatment of stroke through nanotechnology. In: The textbook of Nanoneurosurgery and Nanoneuroscience. Kateb, B, Heiss, J. (eds). CRC Press, 2013 pp 283298.

(12) Tam, R. Y.; Fuehrmann, T.; Mitrousi, N.; Shoichet, M. S. Regenerative Therapies for Central Nervous System Diseases: a Biomaterials Approach. Neuropsychopharmacology 2014, 39 (1), 169-188.

(13) Nguyen, M. K.; Alsberg, E. Bioactive factor delivery strategies from engineered polymer hydrogels for therapeutic medicine. Prog. Polym. Sci. 2014, 39, 1235-1265.

(14) Seib, F. P. Silk hydrogels for drug and cell delivery. In: Hydrogels: Design, Synthesis \& Application in Drug Delivery \& Regenerative Medicine. Singh, T. R. R., Laverty, G, Donnelly, R. (eds) CRC Press, 2018 pp 208-227.

(15) Gasperini, L.; Mano, J. F.; Reis, R. L. Natural polymers for the microencapsulation of cells. J. R. Soc., Interface 2014, 11, 20140817.

(16) Holland, C.; Numata, C.; Rnjak-Kovacina, J.; Seib, P. F. The Biomedical Use of Silk: Past, Present. Adv. Healthcare Mater. 2018, 1800465.

(17) Rockwood, D. N.; Preda, R. C.; Yücel, T.; Wang, X.; Lovett, M. L.; Kaplan, D. L. Materials fabrication from Bombyx mori silk fibroin. Nat. Protoc. 2011, 6, 1612-1631.

(18) Seib, F. P.; Herklotz, M.; Burke, K. A.; Maitz, M. F.; Werner, C.; Kaplan, D. L. Multifunctional silk-heparin biomaterials for vascular tissue engineering applications. Biomaterials 2014, 35 (1), 83-91.

(19) Seib, F. P.; Kaplan, D. L. Silk for drug delivery applications: Opportunities and challenges. Isr. J. Chem. 2013, 53, 1-12.

(20) Osama, I.; Gorenkova, N.; McKittrick, C. M.; Wongpinyochit, T.; Goudie, A.; Seib, P.; Carswell, H. In vitro studies on spaceconforming self-assembling silk hydrogels as a mesenchymal stem cellsupport matrix suitable for minimally invasive brain application. Sci. Rep. 2018, Sep 12, 8 (1), 13655.

(21) Fernández-García, L.; Marí-Buyé, N.; Barios, J. A.; Madurga, R.; Elices, M.; Pérez-Rigueiro, J.; Ramos, M.; Guinea, G. V.; GonzálezNieto, D. Safety and tolerability of silk fibroin hydrogels implanted into the mouse brain. Acta Biomater. 2016, 45, 262-275.

(22) Fernández-García, L.; Pérez-Rigueiro, J.; Martinez-Murillo, R.; Panetsos, F.; Ramos, M.; Guinea, G. V.; González-Nieto, D. Cortical Reshaping and Functional Recovery Induced by Silk Fibroin Hydrogels-Encapsulated Stem Cells Implanted in Stroke Animals. Front. Cell. Neurosci. 2018, 12, 296.

(23) Zhang, Q.; Yan, S.; You, R.; Kaplan, D. L.; Liu, Y.; Qu, J.; Li, X.; Li, M.; Wang, X. Multichannel silk protein/laminin grafts for spinal cord injury repair. J. Biomed. Mater. Res., Part A 2016, 104 (12), 30453057.

(24) Stoppel, W. L.; Hu, D.; Domian, I. J.; Kaplan, D. L.; Black, L. D. Anisotropic silk biomaterials containing cardiac extracellular matrix for cardiac tissue engineering. Biomed. Mater. 2015, 10 (3), 034105.

(25) The, T. K.; Toh, S. L.; Goh, J. C. Aligned hybrid silk scaffold for enhanced differentiation of mesenchymal stem cells into ligament fibroblasts. Tissue Eng. Part C Methods 2011, 17 (6), 687-703.

(26) Leng, X.; Liu, B.; Su, B.; Liang, M.; Shi, L.; Li, S.; Qu, S.; Fu, X.; Liu, Y.; Yao, M.; Kaplan, D. L.; Wang, Y.; Wang, X. In situ ultrasound imaging of silk hydrogel degradation and neovascularization. J. Tissue Eng. Regener. Med. 2017, 11 (3), 822-830.

(27) Seib, F. P. Silk nanoparticles - an emerging anticancer nanomedicine. AIMS Bioengineering 2017, 4 (2), 239-258. 
(28) Kilkenny, C.; Browne, W. J.; Cuthill, I. C.; Emerson, M.; Altman, D. G. Improving bioscience research reporting: the ARRIVE guidelines for reporting animal research. PLoS Biol. 2010, 8, e1000412.

(29) Belayev, L.; Alonso, O. F.; Busto, R.; Zhao, W.; Ginsberg, M. D. Middle cerebral artery occlusion in the rat by intraluminal suture. Neurological and pathological evaluation of an improved model. Stroke 1996, 27 (9), 1616-1622.

(30) Carmichael, S. T. Rodent models of focal stroke: size, mechanism, and purpose. Review. NeuroRx 2005, 2 (3), 396-409.

(31) Lee, S.; Lee, M.; Hong, Y.; Won, J.; Lee, Y.; Kang, S. G.; Chang, K. T.; Hong, Y. Middle cerebral artery occlusion methods in rat versus mouse models of transient focal cerebral ischemic stroke. Neural Regener. Res. 2014, 9 (7), 757-758.

(32) Modo, M.; Stroemer, R. P.; Tang, E.; Patel, S.; Hodges, H. Effects of implantation site of stem cell grafts on behavioral recovery from stroke damage. Stroke 2002, 33 (9), 2270-8.

(33) Modo, M.; Stroemer, R. P.; Tang, E.; Veizovic, T.; Sowniski, P.; Hodges, H. Neurological sequelae and long-term behavioural assessment of rats with transient middle cerebral artery occlusion. J. Neurosci. Methods 2000, 104, 99-109.

(34) Smith, E. J.; Stroemer, R. P.; Gorenkova, N.; Nakajima, M.; Crum, W. R.; Tang, E.; Stevanato, L.; Sinden, J. D.; Modo, M. Implantation site and lesion topology determine efficacy of a human neural stem cell line in a rat model of chronic stroke. Stem Cells 2012, 30 (4), 785-96.

(35) Shearer, J. A.; Coker, S. J.; Carswell, H. V. O. Detrimental effects of 2-arachidonoylglycerol on whole blood platelet aggregation and on cerebral blood flow after a focal ischemic insult in rats. Am. J. Physiol. Heart Circ. Physiol. 2018, 314 (5), H967-H977.

(36) Bederson, J. B.; Pitts, L. H.; Tsuji, M.; Nishimura, M. C.; Davis, R. L.; Bartkowski, H. Rat middle cerebral artery occlusion: evaluation of the model and development of a neurologic examination. Stroke 1986, 17 (3), 472-6.

(37) Anderson, J. M.; Cook, G.; Costerton, B.; Hanson, S. R.; Hensten-Pettersen, A.; Jacobsen, N.; Johnson, R. J.; Mitchell, R. N.; Pasmore, M.; Schoen, F. J.; Shirtliff, M.; Stoodley, P. Host Reactions to Biomaterials and Their Evaluation Testing Biomaterials. In Biomaterials Science. An Introduction to Materials in Medicine, 2nd Ed; Ratner, B, Hoffman, A, Schoen, F., Lemons, F. S. J. (eds). Academic Press, 2004. (38) Liu, W. F.; Ma, M.; Bratlie, K. M.; Dang, T. T.; Langer, R.; Anderson, D. G. Real-time in vivo detection of biomaterial-induced reactive oxygen species. Biomaterials 2011, 32 (7), 1796-1801.

(39) Seib, F. P. Reverse-engineered silk hydrogels for cell and drug delivery. Ther. Delivery 2018, 9 (6), 469-487.

(40) Fox, G.; Gallacher, D.; Shevde, S.; Loftus, J.; Swayne, G. Anatomic variation of the middle cerebral artery in the Sprague-Dawley rat. Stroke 1993, 24, 2087-2092.

(41) Howells, D. W.; Porritt, M. J.; Rewell, S. S.; O'Collins, V.; Sena, E. S.; van der Worp, H. B.; Traystman, R. J.; Macleod, M. R. Different strokes for different folks: the rich diversity of animal models of focal cerebral ischemia. J. Cereb. Blood Flow Metab. 2010, 30 (8), 14121431.

(42) Bettahalli, N. M.; Arkesteijn, I. T.; Wessling, M.; Poot, A. A.; Stamatialis, D. Corrugated round fibers to improve cell adhesion and proliferation in tissue engineering scaffolds. Acta Biomater. 2013, 9 (6), 6928-6935.

(43) Hsieh, W. C.; Chang, C. P.; Lin, S. M. Morphology and characterization of 3D micro-porous structured chitosan scaffolds for tissue engineering. Colloids Surf., B 2007, 57, 250-255.

(44) Cregg, J. M.; DePaul, M. A.; Filous, A. R.; Lang, B. T.; Tran, A.; Silver, J. Functional Regeneration Beyond the Glial Scar. Exp. Neurol. 2014, 253, 197-207.

(45) Venkat, P.; Shen, Y.; Chopp, M.; Chen, J. Cell-based and pharmacological neurorestorative therapies for ischemic stroke. Neuropharmacology 2018, 134, 310-322.

(46) Yiu, G.; He, Z. Glial inhibition of CNS axon regeneration. Nat. Rev. Neurosci. 2006, 7 (8), 617-627.

(47) Catanese, L.; Tarsia, J.; Fisher, M. Acute Ischemic Stroke Therapy. Overview. Circ. Res. 2017, 120, 541-558.
(48) Maysami, S.; Haley, M.; Gorenkova, N.; Krishnan, S.; McColl, B. W.; Lawrence, C. B. Prolonged diet-induced obesity in mice modifies the inflammatory response and leads to worse outcome after stroke. J. Neuroinflammation 2015, 2, 140-152.

(49) Jin, K.; Wang, X.; Xie, L.; Mao, X. O.; Zhu, W.; Wang, Y.; Shen, J.; Mao, Y.; Banwait, S.; Greenberg, D. A. Evidence for stroke-induced neurogenesis in the human brain. Proc. Natl. Acad. Sci. U. S. A. 2006, 103 (35), 13198-13202.

(50) Kazanis, I.; Gorenkova, N.; Zhao, J. W.; Franklin, R. J. M.; Modo, M.; ffrench-Constant, C. The late response of rat subependymal zone stem and progenitor cells to stroke is restricted to directly affected areas of their niche. Exp. Neurol. 2013, 248, 387-97.

(51) Ghuman, H.; Gerwig, M.; Nicholls, F. J.; Liu, J. R.; Donnelly, J.; Badylak, S. F.; Modo, M. Long-term retention of ECM hydrogel after implantation into a sub-acute stroke cavity reduces lesion volume. Acta Biomater. 2017, 63, 50-63.

\section{NOTE ADDED AFTER ASAP PUBLICATION}

This paper was published ASAP on December 11, 2018. Figure 7 was updated. The revised paper was reposted on December 13, 2018. 